\title{
Breeding of potato resistant to late blight using genetic resources and DNA markers
}

\author{
J. Śliwka*, M. Brylińska, E. Stefańczyk, J. Plich, P. Smyda-Dajmund, S. Sobkowiak \\ Plant Breeding and Acclimatization Institute - National Research Institute, Młochów Research Centre, Młochów, Poland
}

DOI 10.18699/ICG-PlantGen2019-69

(c) Autors, 2019

* e-mail: j.sliwka@ihar.edu.pl

\begin{abstract}
Potato (Solanum tuberosum L.) is the fourth most important crop plant worldwide and its economically most important disease, late blight, is caused by an Oomycete, Phytophthora infestans (Mont.) de Bary. Breeding potatoes resistant to the disease is a valid and environment-friendly alternative to the currently applied intensive chemical control. The pathogen is fast-evolving and can quickly adapt and infect new resistant cultivars of the host. Therefore new strategies of using late blight resistance (Rpi) genes in improving durability of the resistance are developed. The history of the search for Rpi genes, their mapping in Plant Breeding and Acclimatization Institute - National Research Institute and our current research are reported here.
\end{abstract}

Key words: potato; Solanum tuberosum L.; late blight resistance; DNA markers.

\section{Introduction}

There are around 240 wild Solanum species that produce tubers and that can be exploited for enrichment of the potato (Solanum tuberosum L.) gene pool (Hawkes 1990). The first important trait that was introduced to the cultivated potato from its wild relatives already in the beginning of the XX century was late blight resistance. The hexaploid wild species Solanum demissum was the source of the first 11 genes for resistance to Phytophthora infestans (Mont.) de Bary (Rpi genes: R1-R11) (Black 1952). The resistance provided by some of those genes $(R 1, R 3, R 4, R 10$ and $R 11)$ that were widely used in potato cultivars soon turned ineffective as new races of $P$. infestans quickly evolved. Since then, many new Rpi genes have been discovered in species such as: S. bulbocastanum, S. pinnatisectum, S. berthaultii, S. microdontum, S. stoloniferum, $S$. venturii and $S$. mochiquense, among others. New strategies have been proposed for applying those genes into practice in ways that should extend the durability of the provided resistance and prevent quick evolution of compatible $P$. infestans strains. Three most important ones are: 1 . pyramiding several Rpi genes in single potato cultivars, 2. growing mixtures of breeding lines/cultivars with different Rpi genes, 3 . growing cultivars with different Rpi genes, or better: gene pyramids, in different growing seasons so the $P$. infestans strains selected to overcome the resistance of a certain gene pyramid would perish in a next season when other Rpi genes are in action. All those strategies are based on the access to multiple Rpi genes and methods for their quick introduction to potato breeding lines such as marker-assisted selection (MAS). Here we present the history of the search for Rpi genes among wild potato relatives, their mapping and exploitation in potato breeding in Plant Breeding and Acclimatization Institute - National Research Institute, Poland.

\section{Materials and methods}

The sources of resistance were:

A hybrid of S. phureja $\times$ S. stenotomum obtained from the International Potato Centre (CIP, Lima, Peru) carrying the Rpi-phul gene (Śliwka et al., 2006, Foster et al., 2009).
Solanum michoacanum (Zoteyeva et al., 2012) obtained from the N.I. Vavilov Institute for Plant Genetic Resources (VIR), (Saint Petersburg, Russia) carrying the Rpi-mchl gene (Śliwka et al., 2012a).

Solanum ruiz-ceballosii Cárd. (Zoteyeva et al., 2012), obtained from the N.I. Vavilov Institute for Plant Genetic Resources (VIR), (Saint Petersburg, Russia), carrying the Rpi-rzcl gene (Śliwka et al., 2012b).

Cultivar Sárpo Mira from Hungarian breeding program, registered in UK in 2002, with at least five genes for late blight resistance: R3a, R3b, R4, Rpi-Smira1 and Rpi-Smira2/ $R 8$ (Tomczyńska et al., 2014).

For screening for resistance and evaluation of mapping populations, detached leaflet, whole tuber and tubers slice tests were performed in laboratory conditions and with standardized inoculum. Selected genotypes were tested in the field under natural infection pressure. Virulence of Polish $P$. infestans population was monitored using detached leaflet tests. For mapping, targeted PCR markers (in the case of the Rpi-phul, Rpi-Smiral genes) or Diversity Array Technology (DArT) markers (Rpi-mchl and Rpi-rzcl) were used. For MAS, closely linked (Rpi-rzcl, Rpi-mch1, Rpi-Smiral) or gene-derived (Rpi-phul, R8) PCR markers were used.

\section{Results and discussion}

The spectrum and durability of resistance provided by different Rpi genes are monitored in the Polish population of $P$. infestans in virulence detached leaflet tests. Each year we collected 100-200 isolates of the pathogen and on the basis of Simple Sequence Repeat (SSR) markers their diversity is assessed and a representative sample is chosen. The results of the virulence tests of 70 P. infestans isolates representative for 2017 are shown in Figure 1. Black's differential R9, differentials with Rpi-phul and Rpi-rzcl genes, as well as the potato cultivars 'Carolus' (Netherlands 2013), 'Alouette' (with the Rpi-vnt1 gene, Netherlands 2014) and 'Gardena' (Poland, 2018) are infected by only a few (0-7) P. infestans isolates, indicating that genes underlying the resistance of those potato genotypes are still useful for breeding purposes. 


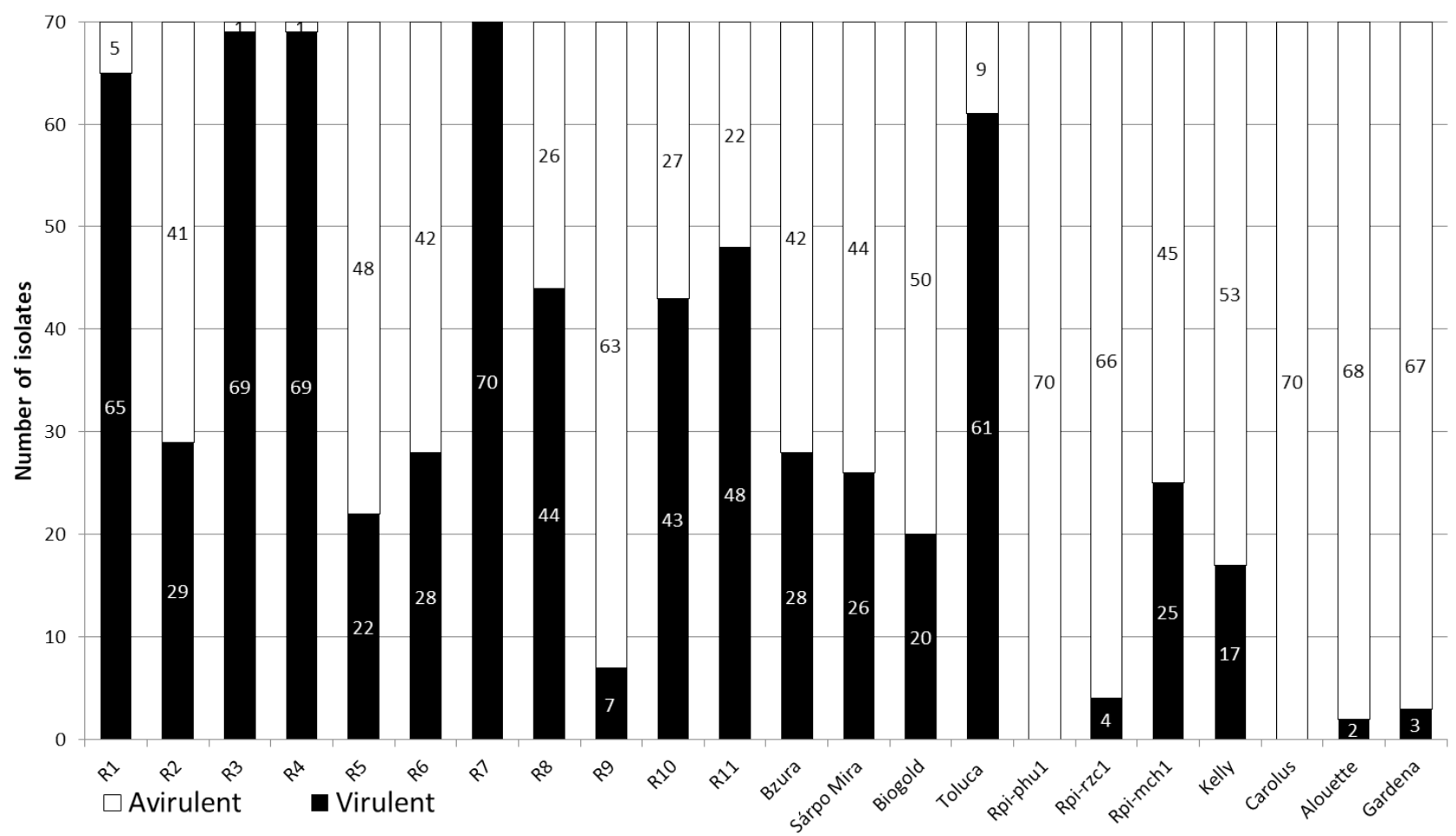

Figure 1. Virulence of 70 representative $P$. infestans isolates collected in Poland in 2017 on Black's differential set (R1-R11), new resistant cultivars and breeding lines carrying the Rpi-phu1, Rpi-rzc1 and Rpi-mch1 genes for late blight resistance. Results of detached leaflet assays.

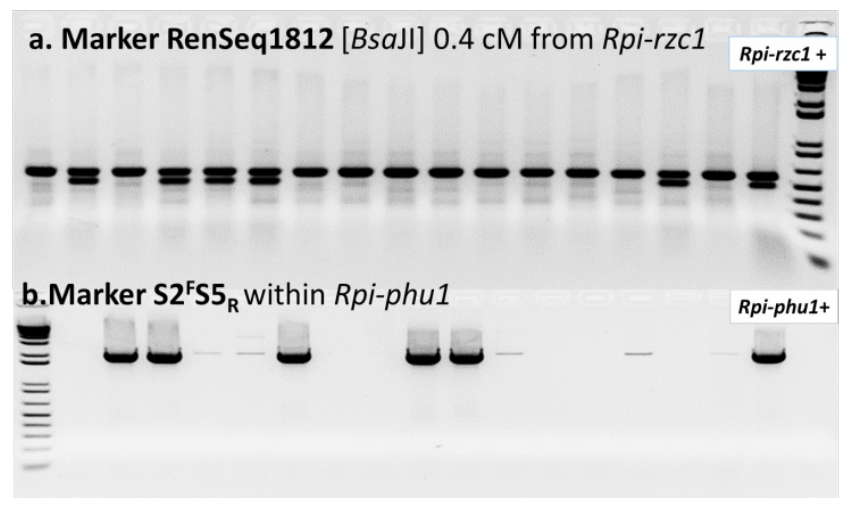

Figure 2. Marker-Assisted Selection of plants with the Rpi-rzc1 and Rpiphu1 genes.

The use of genetic markers such as RenSeq1812 linked to the Rpi-rzcl gene within $0.4 \mathrm{cM}$ (Figure 2,a) or the $\mathrm{S} 2 \mathrm{FS} 5_{\mathrm{R}}$ located within the Rpi-phul gene (Figure 2,b) allows MAS and pyramiding of the resistance genes even when the differential $P$. infestans isolates are not available, as well as it can make the breeding process faster and cheaper. The conventional pre-breeding and breeding process is extremely long when Solanum spp. other than S. tuberosum are used as resistance donors. It is well illustrated by an example of the Rpi-phul gene which was obtained from CIP in 1970, and although we mapped it in 2006 and sequenced it in 2009, only in 2018 the cultivar 'Gardena' carrying that was registered by Zamarte Potato Breeding Ltd. - Group IHAR. The Rpi-phul gene was pyramided with late blight resistance originating from the cultivar 'Sárpo Mira' using MAS for the Rpi-phul gene, Rpi-Smiral (Tomczyńska et al., 2014), and recently also using markers for R8/Rpi-Smira2. To learn more about how Rpi genes function, we performed an expression study in compatible and incompatible interactions between plants with the Rpi-phul gene and P. infestans. The results showed that when avirulent isolates were used, the Rpi-phul expression remained stable and low, while when the virulent isolates were infecting the plants, the expression of the resistance gene was enhanced, which may help the plant to defend itself in field conditions (Stefańczyk et al., 2017).

Our current research focuses on marker-assisted pyramiding of the Rpi-phul and Rpi-rzcl genes at diploid and tetraploid levels. We used three segregating diploid populations to test marker specificities and we performed interploid crosses. The presence of the Rpi-phul homologs in the Rpi-rzcl donor plants resulted in false positive results and required an adjustment of the Rpi-phul gene-derived marker to enhance its specificity.

\section{Conclusions}

The major genes for late blight resistance provide high levels of disease resistance and can be introduced into potato cultivars. Careful choice of effective Rpi genes and gene pyramiding can extend the durability of such resistance. Recently a number of new, highly resistant potato cultivars (e. g. 'Carolus', 'Alouette', 'Gardena') have been registered, offering potato growers an environment-friendly alternative for control of late blight. A conventional breeding process of exploiting various Solanum species is laborious and long. It could be accelerated by transfer of the Rpi genes using genetic modifications, if only this technique were to be broadly accepted. 


\section{References}

Black W. A genetic basis for the classification of strains of Phytophthora infestans. Proceedings of Royal Society B Edinburgh. 1952; 65:36-51.

Foster S.J., Park T.H., Pel M., Brigneti G., Śliwka J., Jagger L., van der Vossen E., Jones J.D. Rpi-vnt1.1, a Tm-2(2) homolog from Solanum venturii, confers resistance to potato late blight. Mol. Plant Microbe Interact. 2009;22:589-600.

Hawkes J.G. The potato, evolution, biodiversity and genetic resources. London: Belhaven Press, 1990.

Stefańczyk E., Sobkowiak S., Brylińska M., Śliwka J. Expression of the potato late blight resistance gene Rpi-phul and Phytophthora infestans effectors in the compatible and incompatible interactions in potato. Phytopathol. 2017;107:740-748.

Śliwka J., Jakuczun H., Lebecka R., Marczewski W., Gebhardt C., Zimnoch-Guzowska E. The novel, major locus Rpi-phul for late blight resistance maps to potato chromosome IX and is not correlated with long vegetation period. Theor. Appl. Gen. 2006;113:685-695.

Śliwka J., Jakuczun H., Chmielarz M., Hara-Skrzypiec A., Tomczyńska I., Kilian A., Zimnoch-Guzowska E. A new resistance gene against potato late blight originating from Solanum $\times$ michoaca- num maps to potato chromosome VII. Theor. Appl. Gen. 2012a;124: 397-406.

Śliwka J., Jakuczun H., Chmielarz M., Hara-Skrzypiec A., Tomczyńska I., Kilian A., Zimnoch-Guzowska E. Late blight resistance gene from Solanum ruiz-ceballosii is located on potato chromosome $\mathrm{X}$ and linked to violet flower colour. BMC Gen. 2012b;13(1):11.

Tomczyńska I., Stefańczyk E., Chmielarz M., Karasiewicz B., Kamiński P., Jones J.D.G., Lees A.K., Śliwka J. A locus conferring effective late blight resistance in potato cultivar Sárpo Mira maps to chromosome XI. Theor. Appl. Gen. 2014;127:647-657.

Zoteyeva N., Chrzanowska M., Flis B., Zimnoch-Guzowska E. Resistance to pathogens of the potato accessions from the collection of N.I. Vavilov Institute of Plant Industry (VIR). Am J Pot Res. 2012; 89:277-293.

Acknowledgements. The research on Rpi-phu1 and Rpi-rzc1 markerassisted pyramiding was funded within the G2P-SOL project (Title: Linking genetic resources, genomes and phenotypes of Solanaceous crops) which has received funding from the European Union's Horizon 2020 research and innovation programme under grant agreement No. 677379.

Conflict of interest. The authors declare no conflict of interest. 